\title{
Dynamic transformations of the internal structure of a moving domain wall in magnetic nanostripes
}

\author{
Jun-Young Lee, Ki-Suk Lee, Sangkook Choi, Konstantin Y. Guslienko, and Sang-Koog Kim* \\ Research Center for Spin Dynamics and Spin-Wave Devices, Seoul National University, Seoul 151-744, Republic of Korea \\ and Nanospintronics Laboratory, Department of Materials Engineering and Science, College of Engineering, Seoul National University, \\ Seoul 151-744, Republic of Korea
}

(Received 19 June 2007; revised manuscript received 19 August 2007; published 8 November 2007)

\begin{abstract}
The magnetic field (or electric current) driven domain-wall motion in magnetic nanostripes is of considerable interest because it is essential to the performance of information-storage and logic devices. One of the current key problems is to understand the complex behaviors of oscillatory domain-wall motions under applied magnetic fields stronger than the so-called Walker field, beyond which the velocity of domain walls markedly drops. In a certain range just above the Walker field, the motions are not chaotic but rather periodic with different periodicities of dynamic transformations of a moving domain wall between the different types of its internal structure. In addition, three different periodicities of the dynamic transformations are calculated, which consist of different types of domain-wall structures that are transformed from one type to another. The transformation periods vary with the field strength and the nanostripe width. This phenomenon can be described by the dynamic motion of a limited number of magnetic topological solitons such as the vortex and antivortex confined in nanostripes. These results provide a considerably better understanding and details of the domainwall motions in soft magnetic nanostripes.
\end{abstract}

DOI: 10.1103/PhysRevB.76.184408

PACS number(s): 75.60.Ch, 75.70.Kw, 75.75.+a

\section{INTRODUCTION}

Magnetic domain walls (DWs) of various internal structures are typically observed in magnetic materials. ${ }^{1-4}$ The DWs are ten- or hundred-nanometer-size transition regions in which the orientation of local magnetizations $\mathbf{M}$ gradually changes between neighboring magnetic domains of different $\mathbf{M}$ orientations in an unsaturated magnetization state. ${ }^{1-3}$ The different structure of DWs varies with material parameters and geometry. In particular, in magnetic films, especially in patterned magnetic films such as stripes, dots, etc., more complicated DW structures appear since magnetostatic interaction is dominant in the restricted geometry. In the case of soft magnetic nanostripes, "head-to-head" or "tail-to-tail" transverse- or vortex-type DWs are present in an equilibrium ground state, as calculated ${ }^{5}$ and observed by various experimental techniques. ${ }^{6-9}$

Recently, the high-velocity propagation, in ferromagnetic nanostripes, of a single DW driven by an applied magnetic field $H$ or spin-polarized current has attracted considerable and growing interest, ${ }^{6-20}$ owing to its crucially important applications to information-storage ${ }^{21,22}$ and logic ${ }^{23}$ devices. It is known that the DW type varies depending on the given width and thickness of the nanostripes, and transverse walls (the DW magnetization has nonzero component transverse to the stripe length) or vortex walls can be stabilized depending on the geometrical parameters. ${ }^{5,13}$ When a static $H$ is applied along the nanostripe length to any type of DW present in the nanostripes (Fig. 1), DW propagates in the $H$ direction to reduce the Zeeman energy $6,7,9,11-14,17,18$ according to the relative orientation between the local $\vec{M}$ and the $H$ direction. The one-dimensional (1D) (one coordinate perpendicular to the DW plane is used) theoretical models were developed to describe DW motion in bulk materials ${ }^{12}$ and magnetic films with strong perpendicular anisotropy (bubble materials). ${ }^{3}$
Within the models, the DW velocity can be described by the simple equation $\nu=\mu H$, where $\mu=\gamma \Delta / \alpha$ is the DW mobility, $\Delta$ is the DW width, $\alpha$ is the Gilbert damping constant, and $\gamma$ is the gyromagnetic ratio. This is true, however, only for cases of relatively low $H$ (linear regime). In stronger fields than a certain threshold field known as the Walker field $H_{W}$, the velocity does not increase but rather rapidly decreases, as demonstrated in several experiments conducted on magnetic nanostripes. ${ }^{9-11,13,14}$ Such a remarkable drop of the timeaveraged velocity $\bar{\nu}$ of DWs moving in nanostripes was

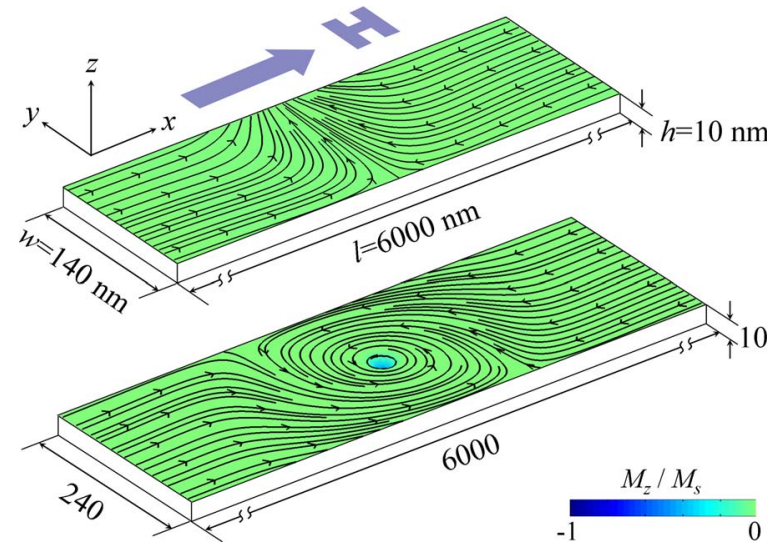

FIG. 1. (Color online) The rectangular-shaped nanostripes of thickness $h=10 \mathrm{~nm}$, length $l=6 \mu \mathrm{m}$, variable widths $w$ as noted, and the coordinate system used. The local magnetization configurations at equilibrium for $w=140$ and $240 \mathrm{~nm}$ display typical headto-head DWs: TW with the V-shaped polarization and VW with the downward core orientation placed at the stripe center. An applied magnetic field, $H$, is indicated by the blue arrow. The streamlines with small arrows on each DW represent the in-plane components of the local magnetization. The spot in the lower panel corresponds to the vortex core. 
known to be associated with the oscillatory motion of DWs, recently. ${ }^{11,14,17,18}$ Although this phenomenon can be qualitatively explained by $1 \mathrm{D}$ model by Schryer and Walker ${ }^{12}$ and some dynamic changes of the internal DW structures in the oscillation regime have been numerically investigated in infinite magnetic films ${ }^{15,16}$ as well as in magnetic nanostripes, ${ }^{11,13,20}$ the underlying physics of the complex oscillatory DW motion has not been unveiled yet in a twodimensional (2D) case such as nanostripes of submicron-size width. We note that, particularly the dynamical transformations of the internal DW structure involving the vortex nucleation on film surface, its propagation along the film thickness and annihilation on the opposite film surface were simulated in Refs. 15 and 16 for infinite films. If we further increase the magnetic field $H$, the oscillation regime will gradually transform to a chaotic (turbulent) regime, and eventually the DW velocity will again increase with the increasing field. The case of magnetic nanostripes is essentially different from the case of infinite films ${ }^{15,16}$ due to the existence of the stripe edges and the nonuniformity of the magnetization distribution in the directions perpendicular to the stripe length and along the length. From the other side, the DW structure can be nonuniform along the film thickness and quite complicated (see Ref. 15 for a recent review on the problem). Thus, understanding the DW motions in a practically applicable 2D nanostripe patterned thin film system is currently a key challenging problem to be solved. ${ }^{10}$ This problem is of special importance in the light of the recent achievements of experimental investigations of the spin-polarized current driven DW motions. ${ }^{17-20}$ The oscillatory DW motion can be excited by current pulses of sufficient strength. ${ }^{17,20}$ Well defined nanosecond oscillations of the nanostripe resistance were observed, which are presumably associated with a periodic change of internal DW structures ${ }^{20}$ but need an adequate physical description.

In this paper, we report various-type oscillatory motions associated with dynamically periodic transformations between different types of internal structures of moving DWs with the characteristic periodicities in the oscillation regime, as studied by micromagnetic calculations on Permalloy $\left(\mathrm{Ni}_{80} \mathrm{Fe}_{20}\right.$; Py) nanostripes of various widths. The DW internal structure transformations and the corresponding periodicities vary with the width of the nanostripes and the strength of the static applied $H$ above $H_{W}$. The results provide a deep understanding of the complex dynamic motions of DWs in the oscillation regime in terms of the emission and absorption of a limited number of moving magnetic topological solitons with integer and fractional topological charges at both edges of the nanostripes. We formulate the problem of the high-speed dynamics of DWs in terms of motion of the magnetic solitons and answer the questions how and why such dynamically periodic transformations occur in the intermediate fields above $H_{W}$.

\section{SIMULATION PROCEDURE}

All micromagnetic simulations in the present study were carried out by procedures similar to those in Refs. 24 and 25, assuming rectangular-shaped Py nanostripes of $10 \mathrm{~nm}$ thick- ness and $6 \mu \mathrm{m}$ total length, as shown in Fig. 1. Different values of the nanostripe width $w$ ranging from 60 to $240 \mathrm{~nm}$ in increments of $20 \mathrm{~nm}$ and different fields $H=5,10,15,20$, and $25 \mathrm{Oe}$, etc., across $H_{W}$ were used. The material parameters corresponding to Py were as follows: the saturation magnetization $M_{s}=8.6 \times 10^{5} \mathrm{~A} / \mathrm{m}$, the exchange constant $A$ $=1.3 \times 10^{-11} \mathrm{~J} / \mathrm{m}$, and zero magnetocrystalline anisotropy. The unit cell dimensions of $5.0 \times 5.0 \times 10.0 \mathrm{~nm}^{3}$ with a constant saturation magnetization for each cell and the Gilbert damping constant $\alpha=0.01$ were used in all of the simulations. The OOMMF code was used to numerically calculate the dynamics of the $\mathbf{M}$ of the individual cells as well as their interactions based on the Landau-Lifshitz-Gilbert equation of motion. ${ }^{25}$ To numerically calculate the dynamic motions of DWs in a nanostripe, we first obtained the equilibrium $\mathbf{M}$ configuration of a head-to-head transverse wall (TW) or vortex wall (VW) type placed at the middle position of the long axis of the nanostripes. These initial $\mathbf{M}$ configurations were obtained with the arbitrary configurations of the TW- or VWlike structures at $H=0$ available at the given widths, according to the simulation results reported in Ref. 13. Our results for the nanostripe thickness of $10 \mathrm{~nm}$ revealed that the static TW (VW) type is stable at $H=0$ below (above) $w_{c}$ $\approx 152 \mathrm{~nm}$, as shown in Fig. 1, in accordance with Ref. 13, where $w_{c}$ is the critical nanostripe width. The static TW- or VW-type M configurations for the given nanostripe width were then driven to move along the nanostripe by a magnetic field applied along the long axis of the nanostripe in the $+x$ direction.

\section{RESULTS AND DISCUSSION}

Upon the application of $H$ along the long axis of the nanostripes, that is, in the $+x$ direction, as noted in Fig. 1, each type of the initial static DW starts to move toward the $\mathbf{H}$ direction because the area of local $\mathbf{M}$, being parallel to the field direction, becomes larger in order to reduce the Zeeman energy. As a result, the movement of the DWs along the nanostripes can be considered as the movement of a classically moving independent object. To determine the DW displacement, we define the time dependent DW position, $\mathbf{X}(t)=[X(t), Y(t)]$, as a point within the nanostripe, where the exchange energy density has a maximum. In the case of VW (or antivortex DW), this point coincides with the extremes of the perpendicular magnetization component, $M_{z}$. We define the instant DW velocity as $\mathbf{v}=d \mathbf{X} / d t$ and the time-averaged DW velocity as $\overline{\mathbf{v}}=T^{-1} \int_{0}^{T} \mathbf{v} d t$, where $T$ is a long enough time interval (the DW oscillation period in our case). Figure 2 shows a remarkable variation of the DW motions in nanostripes as a function of both the parameters $H$ and $w$. Based on the characteristic shapes of the DW displacement versus time (hereafter denoted as $D$ vs $t$ ) curves, the DW characteristic dynamics can be classified, similarly to the 1D case, into two different regimes the linear and oscillatory motions according to the shapes of the linear and oscillatory curves. For the TWs initially stable at $H=0$, its linear motions are observed for the cases of $H$ less than the corresponding value of $H_{W}$ for the given stripe width. However, the VWs initially stable at $H=0$ exhibit slow increases in $\nu$ and then rapid 

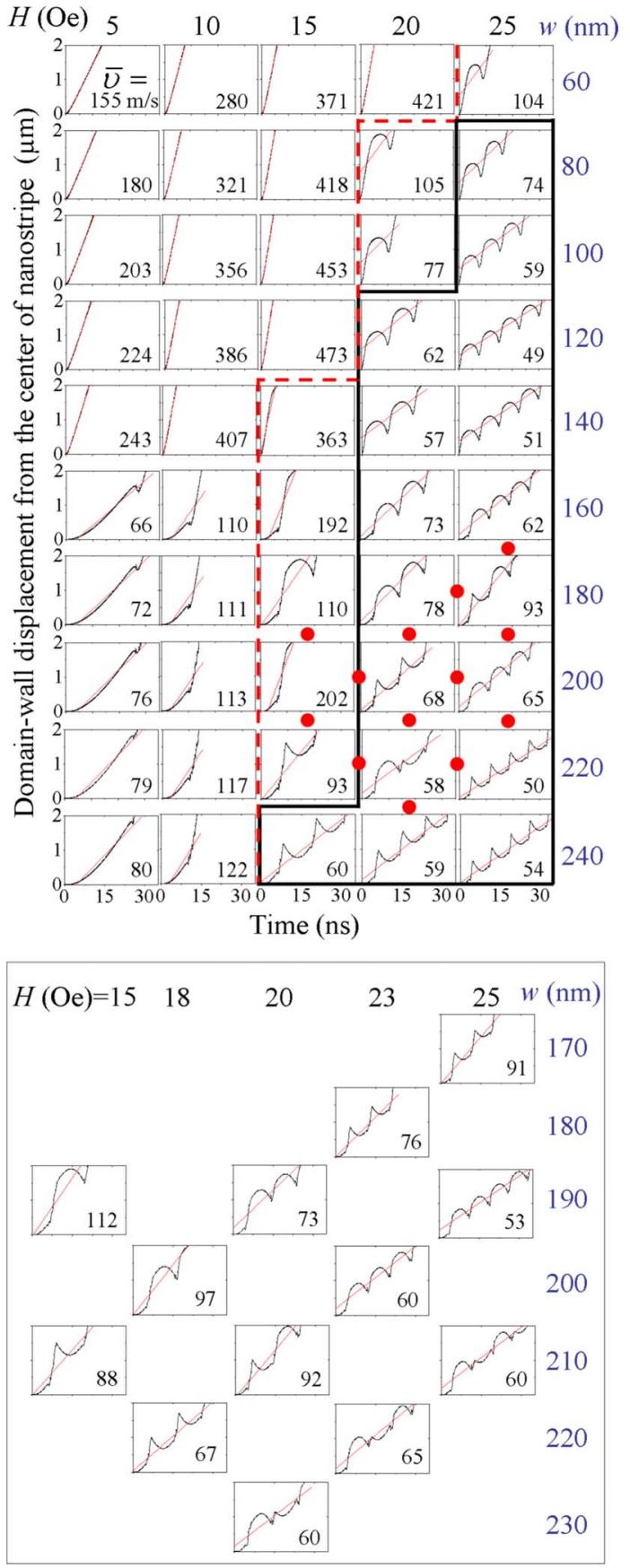

FIG. 2. (Color online) DW displacement versus time curves for the dynamic DW motions along the long axis of nanostripes of $10 \mathrm{~nm}$ thickness, $6 \mu \mathrm{m}$ length, but different widths under the applied static magnetic fields as noted above. Positions of the motions of the individual DW types were plotted by following the vortex or antivortex core positions where the exchange energy had the maximum at each time moment. All the $y$ axes are of the same scale, starting from the middle position of the long axis of the nanostripes and ending at the position $2 \mu \mathrm{m}$ away from the center. All the $x$ axes are of the same scale, ranging from $t=0$ to $35 \mathrm{~ns}$. The red straight line in each box is fitted to the data points within the range of $0-2 \mu \mathrm{m}$, indicating each average velocity $\bar{v}$ for the given fields and widths. Simulations were also carried out for the $w$ and $H$ values marked by the red solid circles, and the results are shown in the bottom panel. The thick dotted red and solid black lines separate the linear (left region) and oscillatory (right region) motion regimes. linear motions from certain onset fields. The initial velocities of the TWs (stable at $H=0$ for $w<w_{c}$ ) are much faster, by several factors, than those of the VWs (for $w>w_{c}$ ), as seen in the comparison of those curves in each column. For example, at $H=5 \mathrm{Oe}$, the values of $\bar{v}$ for the VW and TW types are 80 and $240 \mathrm{~m} / \mathrm{s}$, respectively. For all of the given widths, as $H$ increases, the region where the linear DW motions prevail at an earlier time becomes reduced and, hence, the region where the oscillatory motions occur at a later time becomes larger. The various shapes of the oscillatory $D$ vs $t$ curves represent the various-type oscillatory motions of DWs in the turbulent regime. The oscillatory motions occur in a strictly periodic manner through the entire region of the nanostripes for the cases of $H>H_{W}$ and can be approximately represented as a superposition of linear motion with the velocity $\overline{\mathbf{v}}$ and an oscillatory motion: $\mathbf{X}(t)=\overline{\mathbf{v}} t+\mathbf{X}_{\text {osc }}(t)$. Thus, the velocities of DWs are negative at certain times in a periodic manner, indicating that the DWs sometimes [when the oscillating term $\mathbf{X}_{\text {osc }}(t)$ dominates] move back in the direction opposite to the initial propagation direction (the applied field direction). In addition, the time period of the oscillatory motion $\mathbf{X}_{o s c}(t)$ becomes shorter with further increasing $H$. More interestingly, the shapes of the individual curves in each periodicity differ greatly with changing $H$ and $w$ (see Fig. 2).

Next, the values of $\bar{v}$ for the DWs moving along the nanostripes were plotted versus the field strength $H$ for two cases of $w=140$ and $240 \mathrm{~nm}$, for example, in Fig. 3, which were selected from all of the cases we considered in the simulations (see Fig. 2). The motions of the equilibrium DWs in their initial states are relatively simple at small $H$ values. The linear increase of $\bar{v}$ with increasing $H$ up to $H_{W}$ can be described quite well by a "rigid" DW model. ${ }^{2,3,12}$ However, just above $H_{W}$ (here, approximately $H_{W} \sim 10 \mathrm{Oe}$ ), the $\bar{v}$ decreases remarkably in response to the higher fields, as is known from the literature. ${ }^{2,3,12}$ The two regions are clearly distinguished by the value of $H_{W}$, below and above which the linear and almost constant response of $\bar{v}$ with $H$ is evident. Sometimes, this value of $H_{W}$ and the corresponding maximum velocity are called "bifurcation" field and velocity, respectively. ${ }^{15}$ Also, there is an additional region where $\bar{v}$ increases again with further increase of $H$. Those characteristic DW motions in the three different regions (indicated by I, II, and III) can be briefly described based on the representative snapshot images of the normalized perpendicular magnetization components $M_{z} / M_{s}\left(M_{s}=|\mathbf{M}|\right)$ along with the inplane M orientations for the case of $w=140 \mathrm{~nm}$ (insets of Fig. 3) as well as the $D$ vs $t$ curves for both the cases of $w$ $=140$ and $240 \mathrm{~nm}$.

In region I, a single DW (TW for $w=140 \mathrm{~nm}$ and $\mathrm{VW}$ for $w=240 \mathrm{~nm}$ ) moves steadily along the entire length of the nanostripe. In region II, the oscillatory $D$ vs $t$ curves represent the periodic oscillatory motions of the internal DW structure. This phenomenon is related to the oscillatory transformations of DWs of different types and sequential orders in response to the static external field, as will be addressed in detail later. In region III, the motions of DWs are neither steady nor periodic, but rather chaotic with the appearance of multiple vortex (V) and antivortex (AV) states during the 


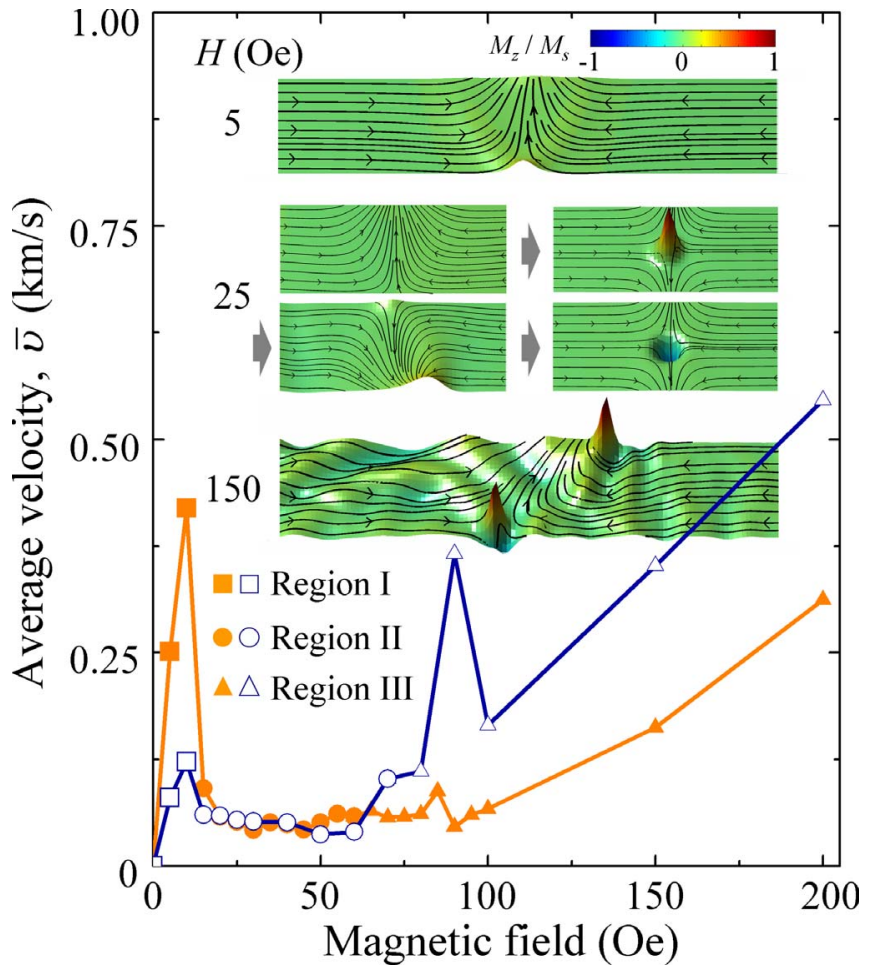

FIG. 3. (Color online) Average velocities of DW motions versus $H$ for two cases of the nanostripe width $w=140 \mathrm{~nm}$ (solid symbols) and $w=240 \mathrm{~nm}$ (open symbols). The square, circle, and triangle symbols for each $w$ case, respectively, indicate region I, the steady motion of a single domain wall, region II, the oscillatory motion of different internal DW structures that are periodically transformed from one to another during the DW motion; and region III, the magnetization reversal via multivortex (multiantivortex) states. The insets show the representative snapshot images of the dynamic internal DW structures in the different characteristic regions for the case of $w=140 \mathrm{~nm}$.

magnetization reversal, which results in much faster average velocity $\bar{v}$ than in region II.

Let us focus on region II, namely, the oscillatory DW motions occurring above $H_{W}$. Similar oscillatory DW dynamics have been explained within the framework of the 1D model in bulk materials, ${ }^{12}$ where the $1 \mathrm{D}$ Bloch wall periodically changes its polarization (the average magnetization in the transverse direction) via the transformation to the Néel wall and back. ${ }^{26}$ Also, the numerical calculations of DW dynamics in $2 \mathrm{D}$ nanostripes ${ }^{20,27,28}$ show that the DW motion is also periodic above $H_{W}$ but that the internal states in the course of DW oscillations are absolutely different from the prediction of Ref. 12. Meanwhile, recent studies have also demonstrated the complexities of the DW dynamic behaviors, ${ }^{11,14-16,20,27-29}$ such as the occurrence of negative differential $\mu$ above $H_{W}$, large contrasts in mobility in low and high magnetic fields, and different $H_{W}$ values depending on the thickness, width, magnetic anisotropy, damping, and edge roughness of nanostripes. ${ }^{11,13,15,29}$ However, our simulation results, more interestingly, reveal not only that the shape of the oscillatory $D$ vs $t$ curves depends on $H$ and $w$, but also that there exist only three different periodicities (within our simulations with all the given values of $w$ and $H$ (a)
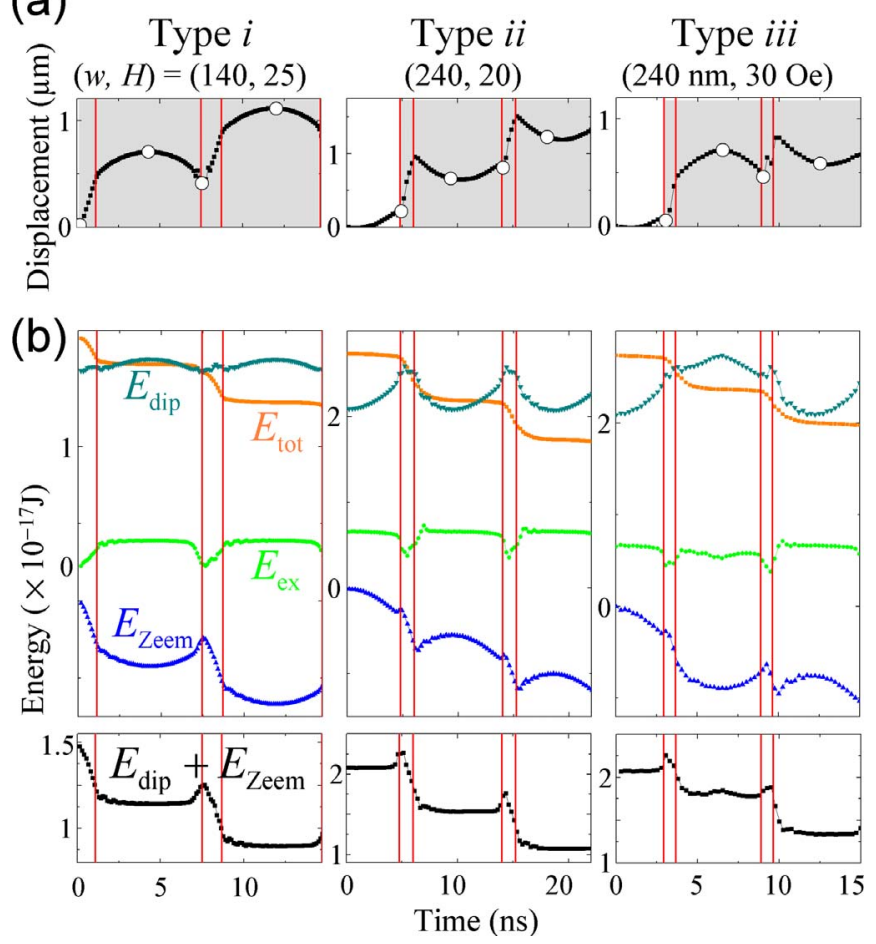

FIG. 4. (Color online) (a) The three different characteristic periods of the oscillatory $D$ vs $t$ curves. (b) Energy variations with time during the periodic DW transformations in the nanostripes. Individual curves indicate the total $E_{\text {tot }}$, Zeeman $E_{\text {Zeem }}$, exchange $E_{\text {ex }}$, and dipolar $E_{\text {dip }}$ energy terms, compared with the sum of $E_{\text {Zeem }}$ and $E_{\text {dip }}$. The vertical red lines separate the individual regions of the DW transformations.

shown in Fig. 2) consisting of the different characteristic shapes of parts of the $D$ vs $t$ curves, including the linear, convex-up, and convex-down curves. The combination of these different shapes determines a variety of the overall $D$ vs $t$ curves (Fig. 2).

To elucidate the underlying physics of how such differenttype oscillatory curves $\mathbf{X}(t)$ occur, we plot in Fig. 4(a) the three different periodicities of the overall shapes of the various $D$ vs $t$ curves along with the variations of the exchange $E_{\text {ex }}$, dipolar $E_{\text {dip }}$, and Zeeman $E_{\text {Zeem }}$ energies of the DW dynamic states, and their sums $\left(E_{\text {tot }}\right.$ and $\left.E_{\text {dip }}+E_{\text {Zeem }}\right)$, as seen in Fig. 4(b). Also, Fig. 5 shows snapshot images representing the detailed instantaneous magnetization configurations of TW, antivortex wall (AVW), and VW types, along with the trajectories of their core motions $\mathbf{X}(t)$ during the periodic oscillatory transformation processes of the corresponding $D$ vs $t$ shapes, as indicated by the gray colors in Fig. 4(a). The trajectories of the motions of the individual DW types were plotted by following the $\mathrm{V}$ or $\mathrm{AV}$ core positions, where the exchange energy has the maximum value at each time moment. We use these positions $\mathbf{X}(t)$ as a convenient definition of coordinates of the corresponding DWs. Here, we chose just three different sets of the parameters $(w, H)$ $=(140 \mathrm{~nm}, 25 \mathrm{Oe}),(240 \mathrm{~nm}, 20 \mathrm{Oe})$, and (240 nm,30 Oe $)$ to show the characteristic periods we found from all the oscillatory shapes of the $D$ vs $t$ curves obtained from our simulations for the given values $w$ and $H$. The distinct periodici- 

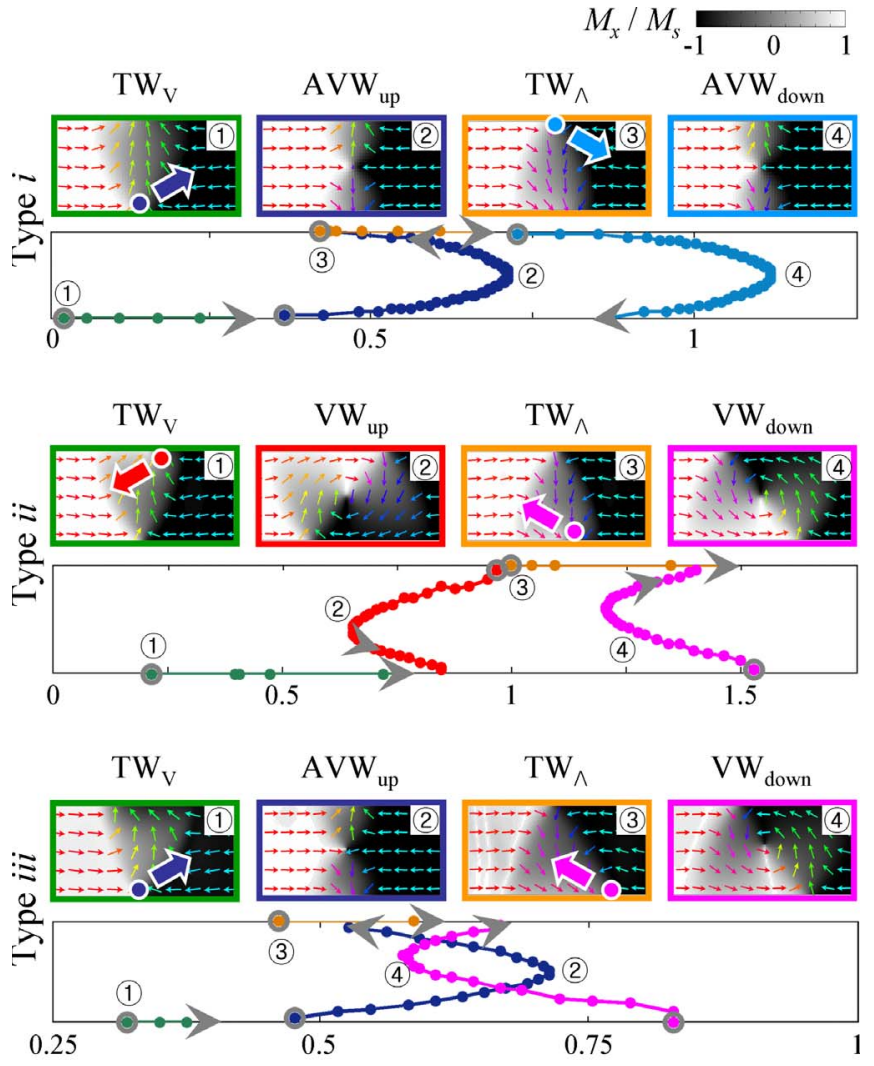

Distance along the long axis $(\mu \mathrm{m})$

FIG. 5. (Color online) Plane-view images of the local in-plane magnetization $\left(M_{x} / M_{s}\right)$ components for the individual internal DW structures in the nanostripes that transform from one type to another in a periodic manner, together with the trajectories of the TW linear motions, and the VW and AVW gyrotropic motions within the nanostripes. The trajectories were plotted by following the maximum exchange energy values at given times [marked in each region and each case indicated by the gray colors in Fig. 4(a)] for the three different cases of $(w, H)=(140 \mathrm{~nm}, 25 \mathrm{Oe}),(240 \mathrm{~nm}, 20 \mathrm{Oe})$, and $(240 \mathrm{~nm}, 30 \mathrm{Oe})$. The solid circles and arrows on the TW types indicate the nucleation sites where the cores of VWs or AVWs are created and the directions of their initial movements, respectively. The arrows on the trajectories indicate the directions of the movement of each DW type. The open gray-colored circles indicate the starting positions of the movements of each DW.

ties are here expressed by types (i), (ii), and (iii) for the sake of convenience. As revealed by the detailed in-plane $\mathbf{M}$ configurations of each type of DW at the given times in the individual regions of the corresponding periodicities, the periodic transformations between the internal DW structures of the TW, AVW, and VW types obviously occur in response to those static fields. The motions of the individual DW types are also represented by the trajectories of their core motions, $\mathbf{X}(t)$. For type (i), the initially stable TW with the V-shaped $\mathbf{M}$ configuration (noted as $\mathrm{TW}_{\mathrm{V}}$ for its polarization) is transformed first to $\mathrm{AVW}_{\text {up }}$, second to $\mathrm{TW}_{\Lambda}$, then to $\mathrm{AVW}_{\text {down }}$, and again to $\mathrm{TW}_{\mathrm{V}}$ itself. The subscripts in each DW type indicate the corresponding polarizations $(\mathrm{V}$ - or $\Lambda$-shaped $\mathbf{M}$ configuration for TW and up- or down-core orientation for either VW or AVW). For type (ii), the $\mathrm{VW}_{\text {down }}$ in the initial state $(H=0)$ is transformed to $\mathrm{TW}_{\mathrm{V}}, \mathrm{VW}_{\mathrm{up}}, \mathrm{TW}_{\Lambda}$, and, again, to $\mathrm{VW}_{\text {down }}$ itself in this sequential order, and hence the dynamic transformation of $\mathrm{TW}_{\mathrm{V}}, \mathrm{VW}_{\mathrm{up}}, \mathrm{TW}_{\Lambda}$, and $\mathrm{VW}_{\text {down }}$ is repeated in this structural change and sequential order as one period. In contrast to these two types, for type (iii), the initial $\mathrm{VW}_{\text {down }}$ is transformed to $\mathrm{TW}_{\mathrm{V}}, \mathrm{AVW}_{\text {up }}, \mathrm{TW}_{\Lambda}$, and $\mathrm{VW}_{\text {down }}$. Quite interestingly, half of each period of types (i) and (ii) appears alternately in one period of type (iii), such as in 1 and 2 of (i) and 3 and 4 of (ii) in this sequence, as seen from Fig. 5. The AVWs or VWs switch their polarizations of either the up- or down-core orientation via their dynamic transformation into the TW type with either polarization. Note that only type (i) was numerically calculated in Ref. 11 without a detailed analysis. The types (ii) and (iii) are calculated in the present work.

Also, the trajectories of the moving cores of the TW, AVW, and VW types in their individual transformation regions clearly exhibit their characteristic motions: the straightforward linear motion for TW, forward and then backward motion for AVW, and vice versa for VW. These characteristic motions of the TW, AVW, and VW types result in the relevant shapes of the sharp increase, convex-up, and convex-down curves, respectively, in the oscillatory $D$ vs $t$ curves (Fig. 2). The AVW and VW types contain a single AV and a single $\mathrm{V}$, respectively, inside the corresponding DWs. The AV and V states bear topological charges (vorticities) ${ }^{30}$ and have nonzero gyrovectors ${ }^{26,31}$ due to the existence of the vortex (antivortex) cores with $\mathbf{M}$ perpendicular to the nanostripe plane. Namely, the nonzero gyrovectors result in their gyrotropic (translation) motion in potential profiles influenced by the total magnetic energy $E_{\mathrm{tot}}(\mathbf{X})$, which contains individual $E_{\mathrm{ex}}, E_{\mathrm{dip}}$, and $E_{\text {Zeem }}$ terms [Fig. 4(b)]. The sense of the gyrotropic motion of $\mathrm{V}$ and $\mathrm{AV}$ in finite-size magnets is determined by the sign of the product of the core polarization $p$ and the soliton topological charge $q$, where $p=+1(-1)$ for the upward (downward) core orientation and $q=+1(-1)$ for $\mathrm{V}(\mathrm{AV}){ }^{31,32}$ Consequently, any type of $\mathrm{V}$ and $\mathrm{AV}$ follows the sense of counterclockwise rotation for $q p=+1$ and clockwise rotation for $q p=-1$ in the gyrotropic motions in a potential well. ${ }^{30-33}$ The principal role of the gyrovectors for the vortex translation motion was demonstrated in Ref. 33 for the case of two-vortex dynamics. Therefore, the $\mathrm{V}$ in the $\mathrm{VW}$ type moves gyrotropically, following its core polarization, as shown in Fig. 5. In contrast, the AV in the AVW type has the topological charge $q=-1$ opposite to the $\mathrm{V}$ topological charge in the VWs $(q=+1)$, such that for the same core polarization and the same kind of the potential well, the gyrotropic motion of AVs should occur in the direction opposite to that of Vs. However, in restricted geometry (nanostripes), the magnetostatic energy $E_{\text {dip }}$ plays a dominant role in forming the potential well for $\mathrm{V}$ or the potential hill for $\mathrm{AV}$ with respect to the middle of the nanostripe [see Fig. 4(b)]. For the nanostripes, the sense of the gyromotion direction is thus determined by the sign of the stiffness coefficient $\kappa$ $=|\kappa| \operatorname{sgn}(q)$ in the corresponding potential profile as well as the product of $p q \cdot^{30,31}$ Therefore, in our case the sense of the $\mathrm{AV}$ or $\mathrm{V}$ gyromotion depends only on the soliton polarization $p$. The AV (V) in the AVW (VW) type rotates gyrotropically counterclockwise for $p=+1$ or clockwise for $p=-1$, which is 
in agreement with the numerical results shown in Fig. 5.

To understand why such periodic transformations occur in the dynamic DW movements, we consider the variations of differently contributing energy terms based on the detailed M configurations of DW types that are transformed from one type to another as well as the trajectories of their cores. For the case of the steady motion of a TW, $E_{\text {dip }}$ and $E_{\text {ex }}$ do not change much (decrease very slowly), but $E_{\text {Zeem }}$ decreases significantly during the straightforward movement along the nanostripe while holding the core of the $\mathrm{TW}_{\mathrm{V}}$ along or nearby the stripe edge in response to $\mathbf{H}$. For moving TWs appearing in the dynamically periodic transformation, $E_{\mathrm{ex}}$ increases markedly, whereas $E_{\text {Zeem }}$ decreases markedly due to their straightforward motions. This occurs because the cores of the AVWs or the VWs continuously move inward (emission of $\mathrm{AV}$ or $\mathrm{V}$ ) the nanostripe according to the nucleation process near the nanostripe edges. Once the cores of the AVWs and VWs are well formed at a distance (about of the core size) far away from the edges, they move inside the nanostripes through their characteristic gyrotropic motions, as mentioned above, in which $E_{\mathrm{ex}}$ does not change much but $E_{\text {Zeem }}$ and $E_{\text {dip }}$ change according to the core positions. As a consequence, $E_{\text {Zeem }}$ for the AVW (VW) type first decreases (increases) and then increases (decreases) because the AVWs (VWs) move first forward (backward) and then backward (forward) through their characteristic gyrotropic motions, as evidenced by their parabolic trajectories shown in Fig. 5. Comparing $E_{\text {dip }}$ of the AVW and VW types, we can state that $E_{\text {dip }}$ for VW (AVW) reaches its minimum (maximum) when the $\mathrm{V}(\mathrm{AV})$ is located in the middle of the nanostripe. Thus, it shows a deep potential well for the VW type and a shallow potential hill for the AVW type. From these energy variations, it can be understood that the increase of $E_{\mathrm{ex}}$ is unavoidable for the nucleation of the cores of VW or AVW inside the nanostripes because the cores bear finite topological charges. To overcome the energy barrier related to this additional $E_{\mathrm{ex}}$ in the further nucleation process, some excess energy should be provided to the nanostripe. Thus in lower fields, TWs continue to move toward the field direction to reduce $E_{\text {Zeem }}$ because the cores of AVW or VW cannot be nucleated inside the nanostripe if the energy barrier is not overcome. However, in higher fields, the cores of AVW or VW can be nucleated near the nanostripe edges by pushing AV (V) inward the nanostripe. Once the cores of the VWs or AVWs are well formed inside the nanostripe, they continue to move inside the nanostripe through the V and AV gyrotropic motions. Note that during the gyrotropic motion of AV or $\mathrm{V}$ after their nucleation, the total energy $E_{\text {tot }}$ and $E_{\mathrm{ex}}$ do not change much; that is, the sum $E_{\text {Zeem }}+E_{\text {dip }}$ is almost constant. The motion with almost constant $E_{\text {tot }}$ corresponds to the V/AV motion along equipotential lines (the damping term is small for Py and can be neglected for the gyromotion) under the influence of the gyroforce, which is perpendicular to the V/AV velocity $\mathbf{v}^{3,26,30}$

Related to the corresponding VW and AVW gyrotropic motions with either up- or down-core orientation, there are obvious correlations of the dynamic transformations between the TW and VW (or AVW) types. The V $(\Lambda$ )-shaped $\mathbf{M}$ configuration of the internal structure of TWs always leads to the upward (downward)-core orientation of the VWs or
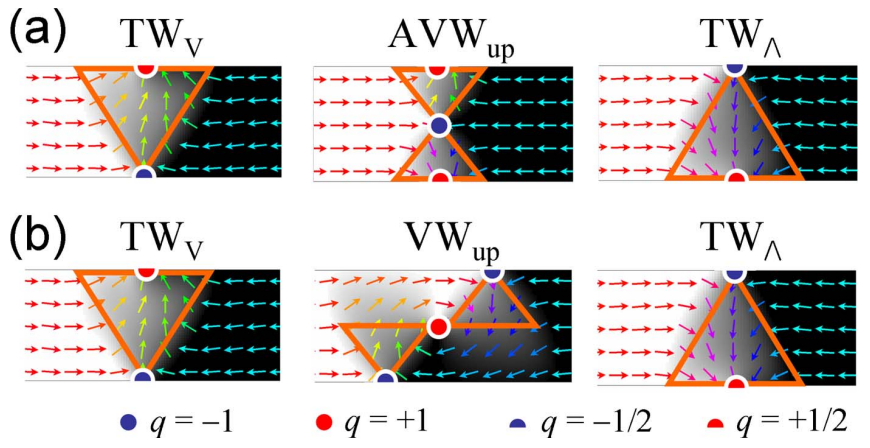

FIG. 6. (Color online) Half-integer and integer topological charges $(q)$ of each type of DWs in the nanostripes represented as magnetic solitons. The topological charges within each DW are indicated by the open and solid circles (semicircles), as noted. The sum of the topological charges of each DW is always zero. The solid-line triangles on the TW, VW, and AVW structures indicate schematically the local magnetization configurations of the head-tohead DWs.

AVWs to be transformed from the TWs themselves due to the rotation sense of the $\mathrm{V}$ and $\mathrm{AV}$ gyrotropic motions, which is determined by $p$ only. Thus, the VW (or AVW) switches its up- and down-core polarization alternatively through the transformation to the $\mathrm{TW}_{\mathrm{V}}$ and $\mathrm{TW}_{\Lambda}$ type alternatively as well.

The nucleation sites at either stripe edges, where the cores of TWs with $\mathrm{V}$ - or $\Lambda$-shaped $\mathbf{M}$ configuration are located, also determine whether the VW or AVW type will be created in the next transformation process. The in-plane $\mathbf{M}$ configuration of the TWs resembles an isosceles triangle with three apexes (see Fig. 6). For the $\mathrm{V}(\Lambda)$-shaped polarization, a single apex is located at the bottom (top) edge, whereas the double apexes at the other side are located at the top (bottom) edge. When the single apexes act as the nucleation sites of the cores to be created in the next transformation, the AVW type is always formed. When one of the other double apexes at the same edge side acts as the nucleation sites for the cores, the VW type is always created. These correlations are associated with the rotation sense of the V and AV gyromotions and the given polarization state of the TW type that is ready to be transformed to the $\mathrm{VW}$ or AVW type. The $\mathrm{V}$ and AV gyromotions are relatively slow and lead to negative DW velocities due to their characteristic backward motions against the field direction. This behavior, in turn, leads to the essential decrease of the average DW velocity.

The drastic dynamic changes of the internal DW structures of different types, described above, are nontrivial. Such periodic DW transformations occurring above $H_{W}$ can be described in terms of the nucleation and annihilation of $\mathrm{V}$ or $\mathrm{AV}$ at either edge of nanostripes of a certain narrow width, that is, the emission and absorption of topological magnetic solitons. The qualitative description of these processes in nanostripes can be done within a general topological soliton approach to thin soft magnetic elements developed by Guslienko and Metlov. ${ }^{34}$ This approach in the form of the $X Y$ model with simplified magnetostatics was then applied to magnetic nanostripes and nanorings in Ref. 35. The Vs and AVs have such topological charges as $q=+1$ and $q=-1$, re- 
spectively, as mentioned before. In contrast, the TWs have nonzero average transverse magnetizations, directed "upward" $\left(\mathrm{TW}_{\mathrm{V}}\right)$ or "downward" $\left(\mathrm{TW}_{\Lambda}\right)$, which was already represented by the V- or $\Lambda$-shaped $\mathbf{M}$ configuration in the nanostripe plane. These configurations lead to half-integer $(q= \pm 1 / 2)$ topological solitons located on the stripe edges (see Fig. 6) in the sense that only half of the core is located inside the nanostripe ${ }^{34}$ (see also the definition of the topological charges via the winding numbers in Ref. 35). The AVWs and VWs have, except the topological charges related to the $\mathrm{AV}$ and $\mathrm{V}$ cores, respectively, also some half-integer charges due to their edge singularities similar to the ones in the TWs, as indicated in Fig. 6. The total topological charge $Q$ of all of the solitons (indexed by $j$ ) inside the nanostripe does not change in the course of their motion that is, $Q$ $=\Sigma_{j} q_{j}=$ const $(Q=0$ for the present model $)$ in the process of the dynamic transformation of each type of DWs. The single apex of the V-shaped TW configuration corresponds to a half-integer topological charge $q=-1 / 2$ (only half of the soliton core is inside the nanostripe). Through such soliton, only the AV can be nucleated with $q=-1$, changing the edge soliton topological charge from $-1 / 2$ to $+1 / 2$. The emitted $\mathrm{AV}$ moves gyrotropically (see the AV trajectory in Fig. 5, first row) to the opposite stripe edge, where the edge soliton adsorbs the $\mathrm{AV}$, changing its topological charge from $+1 / 2$ to $-1 / 2$. The double-apex nucleation site corresponds to the edge soliton with $q=+1 / 2$, and only the vortex with $q=+1$ can be nucleated, changing the edge soliton topological charge from $+1 / 2$ to $-1 / 2$ (see Fig. 6). The emitted V moves (see Fig. 5, second row) to the opposite stripe edge, where the edge soliton adsorbs the $\mathrm{V}$, changing its charge from $-1 / 2$ to $+1 / 2$. The slow motion of the $\mathrm{Vs}$ and $\mathrm{AVs}$ between the occasions of their emission and absorption on the stripe edges determines the period of the DW oscillations. Note that to describe properly the dynamical transformation of the DW internal structure by means of the motion of several topological magnetic solitons, we need to introduce the soliton (V and $\mathrm{AV})$ cores and corresponding gyrovectors. That cannot be done within the two-dimensional $X Y$ model assuming an in-plane magnetization distribution. Nevertheless, the $X Y$ model can be used in the static case for the classification of solitons as "topological defects" of the magnetization field in flat nanomagnets. ${ }^{35}$

\section{CONCLUSIONS}

The present micromagnetic simulations of the field driven DW dynamics in magnetic nanostripes of various widths and the qualitative interpretation of the observed periodic transformations of different-type DWs offer a better understanding of the complex behavior of oscillatory DW motion. The various types of the oscillatory motion are determined by the types of dynamic changes of the internal structures of different DWs, which have the three different periodicities. The overall dynamic motion proceeds through the nucleation, gyrotropic motion, and annihilation of magnetic Vs and AVs in different characteristic periodic manners. This behavior can be described in terms of the emission and absorption of the magnetic topological solitons (Vs and AVs) by the edge solitons above a threshold field by following the conservation of the total topological charge in the nanostripes. Accordingly, the characteristic motions of DWs in the oscillation regime are thus caused not only by the periodic transformations of internal DW structures from the TW type to the AVW or VW type, and back, but also by the backward and forward motion for the VWs or vice versa for the AVWs due to their gyrotropic motion, which gives rise to a significant reduction in the DW average velocities. It is demonstrated that the singleor double-apex nucleation site of the V- or $\Lambda$-shaped TWs determines the emitted soliton charge $q$, whereas the energy conservation [the shape of the magnetostatic well (hill)] determines the soliton core polarization $p$ and the direction of their further gyrotropic motion.

The obtained results not only offer a physical understanding of the complex DW dynamics in patterned thin films, but also serve as a nontrivial example of the strictly periodic dynamical response of a nonlinear system to a steady external perturbation.

\section{ACKNOWLEDGMENTS}

This work was supported by the Creative Research Initiatives (Research Center for Spin Dynamics and Spin-Wave Devices) of MOST/KOSEF. We are grateful to K. L. Metlov and $\mathrm{O}$. Tchernyshyov for valuable discussions.
*Author to whom correspondence should be addressed; sangkoog@snu.ac.kr

${ }^{1}$ C. Kittel, Rev. Mod. Phys. 21, 541 (1949).

${ }^{2}$ A. Hubert and R. Schafer, Magnetic Domains: The Analysis of Magnetic Microstructures (Springer, Berlin, 1998).

${ }^{3}$ A. Malozemoff and J. Slonczewski, Magnetic Domain Walls in Bubble Materials (Academic, New York, 1979).

${ }^{4}$ S.-K. Kim, J. B. Kortright, and S.-C. Shin, Appl. Phys. Lett. 78, 2742 (2001).

${ }^{5}$ R. D. McMichael and M. J. Donahue, IEEE Trans. Magn. 33, 4167 (1997).

${ }^{6}$ T. Ono, H. Miyajima, K. Shigeto, K. Mibu, N. Hosoito, and T.
Shinjo, Science 284, 468 (1999).

${ }^{7}$ D. Atkinson, D. A. Allwood, G. Xiong, M. D. Cooke, C. C. Faulkner, and R. P. Cowburn, Nat. Mater. 2, 85 (2003).

${ }^{8}$ L. Thomas, C. Rettner, M. Hayashi, M. G. Samant, S. S. P. Parkin, A. Doran, and A. Scholl, Appl. Phys. Lett. 87, 262501 (2005).

${ }^{9}$ G. S. D. Beach, C. Nistor, C. Knutson, M. Tsoi, and J. L. Erskine, Nat. Mater. 4, 741 (2005).

${ }^{10}$ R. Cowburn and D. Petit, Nat. Mater. 4, 721 (2005).

${ }^{11}$ Y. Nakatani, A. Thiaville, and J. Miltat, Nat. Mater. 2, 521 (2003).

${ }^{12}$ N. L. Schryer and L. R. Walker, J. Appl. Phys. 45, 5406 (1974). 
${ }^{13}$ Y. Nakatani, A. Thiaville, and J. Miltat, J. Magn. Magn. Mater. 290-291, 750 (2005).

${ }^{14}$ A. Kunz, J. Appl. Phys. 99, $08 \mathrm{G} 107$ (2006).

${ }^{15}$ B. N. Filippov, Low Temp. Phys. 28, 707 (2002).

${ }^{16}$ S. W. Yuan and H. N. Bertram, Phys. Rev. B 44, 12395 (1991).

${ }^{17}$ L. Tomas, M. Hayashi, X. Jiang, R. Moriya, C. Rettner, and S. P. Parkin, Nature (London) 443, 197 (2006).

${ }^{18}$ M. Laufenberg, W. Bührer, D. Bedau, P.-E. Melchy, M. Kläui, L. Vila, G. Faini, C. A. F. Vaz, J. A. C. Bland, and U. Rüdiger, Phys. Rev. Lett. 97, 046602 (2006).

${ }^{19}$ M. Hayashi, L. Thomas, C. Rettner, R. Moriya, X. Jiang, and S. P. P. Parkin, Phys. Rev. Lett. 97, 207205 (2006).

${ }^{20}$ M. Hayashi, L. Tomas, C. Rettner, R. Moriya, and S. S. P. Parkin, Nat. Phys. 3, 21 (2007).

${ }^{21}$ M. Tsoi, R. E. Fontana, and S. S. P. Parkin, Appl. Phys. Lett. 83, 2617 (2003).

${ }^{22}$ J. Grollier, P. Boulenc, V. Cors, A. Hamzić, A. Vaurès, and A. Fert, Appl. Phys. Lett. 83, 509 (2003).

${ }^{23}$ D. A. Allwood, G. Xiong, M. D. Cooke, C. C. Faulkner, D. Atkinson, N. Vernier, and R. P. Cowburn, Science 296, 2003 (2002).

${ }^{24}$ J.-Y. Lee, K.-S. Lee, and S.-K. Kim, Appl. Phys. Lett. 91, 122513 (2007).
${ }^{25}$ K.-S. Lee, S. Choi, and S.-K. Kim, Appl. Phys. Lett. 87, 192502 (2005).

${ }^{26}$ A. A. Thiele, J. Appl. Phys. 45, 377 (1974).

${ }^{27}$ J. He, Z. Li, and S. Zhang, Phys. Rev. B 73, 184408 (2006).

${ }^{28}$ A. Thiaville, J. M. Garcia, and J. Miltat, J. Magn. Magn. Mater. 242-245, 1061 (2002).

${ }^{29}$ B. N. Filippov, L. G. Korzunin, and F. A. Kassan-Ogly, Phys. Rev. B 64, 104412 (2001).

${ }^{30}$ K. Y. Guslienko, B. A. Ivanov, V. Novosad, Y. Otani, H. Shima, and K. Fukamichi, J. Appl. Phys. 91, 8037 (2002).

${ }^{31}$ K. Y. Guslienko, X. F. Han, D. J. Keavney, R. Divan, and S. D. Bader, Phys. Rev. Lett. 96, 067205 (2006).

${ }^{32}$ S.-B. Choe, Y. Acremann, A. Scholl, A. Bauer, A. Doran, J. Stöhr, and H. A. Padmore, Science 304, 420 (2004).

${ }^{33}$ K. Buchanan, P. Roy, M. Grimsditch, F. Fradin, K. Y. Guslienko, S. D. Bader, and V. Novosad, Nat. Phys. 1, 172 (2005).

${ }^{34}$ K. Y. Guslienko and K. L. Metlov, Phys. Rev. B 63, 100403 (2001); K. Y. Guslienko, V. Novosad, Y. Otani, H. Shima, and K. Fukamichi, ibid. 65, 024414 (2002); K. L. Metlov, Phys. Status Solidi A 189, 1015 (2002).

${ }^{35}$ O. Tchernyshyov and G.-W. Chern, Phys. Rev. Lett. 95, 197204 (2005); G.-W. Chern, H. Youk, and O. Tchernyshyov, J. Appl. Phys. 99, 08Q505 (2006). 